\title{
A THTEE-DIMENSIONAL VARIATIONAL ASSIMILATION SCHEME FOR SATELLITE AOD
}

\author{
Yanfei Liang ${ }^{1}$, Zengliang Zang ${ }^{1, *}$, Wei You ${ }^{1}$ \\ ${ }^{1}$ Institute of Meteorology and Oceanography, National University of defense technology, China
}

\author{
Commission VI, WG III/8
}

KEY WORDS: AOD, Aerosol, Data assimilation, Chemical species, WRF/Chem

\begin{abstract}
:
A three-dimensional variational data assimilation scheme is designed for satellite AOD based on the IMPROVE (Interagency Monitoring of Protected Visual Environments) equation. The observation operator that simulates AOD from the control variables is established by the IMPROVE equation. All of the 16 control variables in the assimilation scheme are the mass concentrations of aerosol species from the Model for Simulation Aerosol Interactions and Chemistry scheme, so as to take advantage of this scheme in providing comprehensive analyses of species concentrations and size distributions as well as be calculating efficiently. The assimilation scheme can save computational resources as the IMPROVE equation is a quadratic equation. A single-point observation experiment shows that the information from the single-point AOD is effectively spread horizontally and vertically.
\end{abstract}

\section{INTRODUCTION}

Aerosols are airborne suspensions of minute particles and have fundamental impacts on the earth's environment and climate and on human health. To make a better understand of the chemical and dynamical processes associated with aerosols, many observing networks have been established giving us abundant data about aerosols, including in-situ surface measurements of aerosol mass concentration and remote sensing aerosol optical depth(AOD) (Diner et al., 2004a), and a variety of atmospheric chemistry models have been developed (Fast et al., 2006a).

Aerosol data assimilation is very important for making fully use of the abundant data and improving the prediction effect of aerosol forecasting model. Using one of the many different methods of data assimilation, the different kinds of data such as the satellite AOD and the surface aerosol concentration can be combined to produce optimal estimation of aerosol fields and to improve the prediction effect of aerosol forecasting model. In the last decades, data assimilation has increasingly been applied to aerosol analysis ( $\mathrm{Li}$ et al., 2012a).

Here we present a three-dimensional variational data assimilation (3-DVAR) scheme for satellite AOD based on the IMPROVE (Interagency Monitoring of Protected Visual Environments) equation. This 3-DVAR scheme is developed for the Weather Research and Forecasting (WRF) model coupled with Chemistry (WRF/Chem) (Grell et al., 2005a), with the comprehensive aerosol scheme known as the Model for Simulating Aerosol Interactions and Chemistry (MOSAIC) (Zaveri et al., 2008a). MOSAIC was first implemented in the WRF/Chem by Fast et al (2006a), and has been applied to show the multi-species concentrations and size distributions information of aerosol. The IMPROVE equation is a simple method of calculating the extinction coefficient of aerosols
(Pitchford et al., 2012a). Using the IMPROVE equation can save large amount of computing resources for the calculation of the complicated observation operator of AOD.

Following is the outline of this paper: in Sect. 2, briefly describe the basic formulation of 3-DVAR. In Sect. 3, describe the more challenges faced in AOD assimilation than in the routine observation data assimilation such as the aerosol mass concentration and the strategies for meeting those challenges. In Sect. 4, give a brief description of the MOSAIC scheme; and define the control variables used in the 3-DVAR scheme, Sect. 5 , a single-point observation experiment is performed to test the ability of the assimilation scheme. Finally, in Sect. 6, give a summary and discussion.

\section{BASIC FORMULATION OF 3-DVAR}

The 3-DVAR cost function is written as( $\mathrm{Li}$ et al., 2008a):

$$
\mathrm{J}(x)=\frac{1}{2}\left(x-x^{b}\right)^{T} B^{-1}\left(x-x^{b}\right)+\frac{1}{2}(H x-y)^{T} R^{-1}(H x-y)
$$

Here $\boldsymbol{x}$ is the $\mathrm{N}$ vector, known as the control variable, or state variable, $x^{b}$ is the background or forecast state of the control variable generated in the WRF/Chem with certain aerosol scheme, MOSAIC scheme in this paper. $\mathbf{B}$ is the $\mathrm{N} \times \mathrm{N}$ matrix, denoting the error covariance associated with $x^{b}$. The $(H x-y)$ is a $\mathrm{M}$ vector known as the observation innovation, where $\boldsymbol{y}$ is an observation vector, and the $\mathrm{M} \times \mathrm{N}$ matrix $\mathbf{H}$ is an observational operator that transforms the model control vector into the same parameters as the observation. $\mathbf{R}$ is the $\mathrm{M} \times \mathrm{M}$ matrix denoting the observation error covariance associated with the observation $y$. The minimization solution is the analysis $x^{a}$ which is statistically optimal as a minimum error variance estimate (Jazwinski, 1970) or a maximum likelihood (Bayesian) estimate if both forecast and observation errors have

* Zengliang Zang, zzlqxxy@163.com

This research was supported by the National Natural Science Foundation of China(41775123) 
Gaussian distributions.

For the convenience of the numerical calculation, the incremental form of the 3-DVAR cost function is written as:

$$
\mathrm{J}(\delta x)=\frac{1}{2}(\delta x)^{T} B^{-1}(\delta x)+\frac{1}{2}(H \delta x-d)^{T} R^{-1}(H \delta x-d)
$$

Here $\delta x$ is the incremental control variable, which is defined as $\delta x=\left(x-x^{b}\right)$. The $\mathrm{M}$ vector $d=\left(y-H x^{b}\right)$ is the observation innovation. The minimisation solution is the so-called analysis increment $\delta x^{a}$, and the final analysis is $x^{a}=\left(\delta x^{a}+x^{b}\right)$.

\section{CHALLENGES FACED IN AOD ASSIMILATION AND THE STRATEGIES}

There are some more challenges in AOD assimilation than in the aerosol mass concentration data assimilation. The firs challenge is associated with the observational operator $\mathbf{H} . \mathbf{H}$ is a $\mathrm{M} \times \mathrm{N}$ matrix that maps the control variable to the observation AOD. It is assumed to be linear in the basic formulation of data assimilation, but this assumption cannot be satisfied in AOD assimilation. Tangent linear coding and testing can solve the problem, but the complicity of the tangent linear coding depends on the complicity of $\mathbf{H}$. And the adjoint coding and testing following the tangent linear coding is even more complicated. The CRTM (Community Radiative Transfer Model) or the other radiative transfer model are usually as the observation operators. But the calculation of these observation operators spends a large amount of computing resources for the complicated calculation of Mie scattering.

Another challenge is the large number of control variables. Data assimilation is computationally demanding, although the 3DVAR is relatively computationally efficient among the widely used data assimilation schemes, the computational resources needed in the scheme increase rapidly as the number of control variables increases. A sophisticated model may treat more than a dozen species, each specie has a range of aerosol size distributions ranging from a few nanometers to around $100 \mu \mathrm{m}$ in diameter. So the number of control variables should be designed properly.

The first challenge could be solved by applying the IMPROVE equation. The IMPROVE equation is a simple method of calculating the extinction coefficient of aerosols. Using the IMPROVE equation, the extinction coefficients of different aerosol species can be calculated. Then the AOD over the entire atmosphere can be calculated by integrating the aerosol extinction coefficient vertically. The IMPROVE algorithm for estimating light extinction was adopted by the U.S. Environmental Protection Agency (EPA) as the basis for the regional haze metric used to track progress in reducing haze levels for visibility protected areas under the 1999 Regional Haze Rule (RHR).3, and has been proven to be a useful tool for assessing the impact of specific types of aerosols on visibility(Kim et al., 2006a). Here we use the revised IMPROVE algorithm, which significantly reduces the biases compared with measurements at the high and low extremes, compared with the original algorithm. The IMPROVE equation for aerosol light extinction is as following:

$$
\begin{aligned}
& \text { bet }_{\text {ext }} \approx 2.2 \times f s(R H) \times[\text { Small } \quad \text { Sulfate }]+ \\
& 4.8 \times f l(R H) \times\left[\begin{array}{ll}
\operatorname{Larg} e & \text { Sulfate }]+
\end{array}\right. \\
& 2.4 \times f s(R H) \times[\text { Small Nitrate }]+ \\
& 5.1 \times f l(R H) \times[L \operatorname{larg} e \quad \text { Nitrate }]+ \\
& 2.8 \times[\text { Small Organic Mass }]+ \\
& 6.1 \times\left[\begin{array}{lll}
\text { Large } & \text { Organic Mass }]+
\end{array}\right.
\end{aligned}
$$

$$
\begin{aligned}
& 10 \times[\text { Elemental Carbon }]+ \\
& 1 \times[\text { Fine Soil }]+ \\
& 1.7 \times f \text { ss }(\text { RH }) \times[\text { Sea Salt }]+ \\
& 0.6 \times[\text { Coarse Mass }]
\end{aligned}
$$

The units for light extinction is inverse megameters $\left(10^{-6} \mathrm{~m}\right.$ usually written $\mathrm{Mm}^{-1}$ ), component concentrations shown in brackets are in microgram per meter cubed $\left(\mu \mathrm{g} / \mathrm{m}^{3}\right)$; dry mass extinction efficiency terms are in units of meters squared per $\operatorname{gram}\left(\mathrm{m}^{2} / \mathrm{g}\right)$; and the water growth terms, $f(R H)$, are unitless. The observational operator $\mathbf{H}$ is done by transforming the aerosol species used in the IMPROVE equation from the control variables. And the tangent linear coding will be rather simple since the equation is quadratic equation of the control variables.

The strategy for meeting the second is associated with both the observational operator and the aerosol scheme applied in the atmospheric chemistry models. Another limitation is the computational resources. In this paper, 16 control variables are designed, each of which is transformed from the MOSAIC scheme of WRF/Chem, and are easily applied to the IMPROVE equation. The details of the control variables will be introduced in the following chapter.

\section{MOSAIC SCHEME AND THE CONTROL VARIABLES USED IN THE 3-DVAR SCHEME}

MOSAIC was one of the aerosol chemistry options in WRFchem. It treats eight major species including elemental/black carbon $(E C / B C)$, organic carbon $(O C)$, nitrate $\left(\mathrm{NO}_{3}^{-}\right)$, sulfate( $\left.\mathrm{SO}_{4}^{2-}\right), \quad$ chloride $\left(\mathrm{CL}^{-}\right), \quad \operatorname{sodium}\left(\mathrm{NA}^{+}\right)$, ammonium( $\mathrm{NH}_{4}^{+}$), and the sum of other inorganic, inert mineral and metal species $(O I N)$. The aerosol size distribution is divided into discrete size bins. The bins are defined by their lower and upper dry particle diameters. Each bin is assumed to be internally mixed so that all particles within a bin are assumed to have the same chemical composition. The number of size bins is flexible and can be specified as appropriate for different applications. In this paper 4 bins are used(see Table 1). There are thus 32 aerosol model variables in the MOSAIC model.

\begin{tabular}{|c|c|c|}
\hline Bin & Lower Diameter, $\mu m$ & Upper Diameter, $\mu m$ \\
\hline 1 & 0.039 & 0.1 \\
2 & 0.1 & 1.0 \\
3 & 1.0 & 2.5 \\
4 & 2.5 & 10.0 \\
\hline
\end{tabular}

Table.1 Particle Dry-Diameter Range for the Four Size Bins Employed by MOSAIC in This Paper

The number of control variable could not be as large as the num of model variables in the MOSAIC model(32) considering the computational resources. As the upper diameter of all the components but the Coarse Mass in the IMPROVE equation are $2.5 \mu \mathrm{m}$, we designed 16 control variables. The No.1-No.8 control variables include the sum of the mass concentrations of the first three bins for each aerosol species, and the No.9-No.16 control variables include the mass concentration of the last bin for each aerosol species. This scheme can take advantage of the MOSAIC scheme in providing comprehensive analyses of species concentrations and size distributions as well as be calculating efficiently. 


\section{SINGLE-POINT OBSERVATION EXPERIMENT}

forecasting model are all 0 , and there is an AOD observation with a value of 3.4 in $115.6 \mathrm{E}^{\circ}, 39 \mathrm{~N}^{\circ}$. After the data assimilation, the optimal estimated mass concentrations of 7 of the control variables in the initial field are as showed in figure 1 to figure 7. The estimated mass concentrations of the rest control variables are not showed because of their tiny concentration values. All figures show the vertical distribution of mass concentrations in $115.6 \mathrm{E}^{\circ}, 39 \mathrm{~N}^{\circ}$. Results showed that the information from the single-point AOD is effectively spread horizontally and vertically.

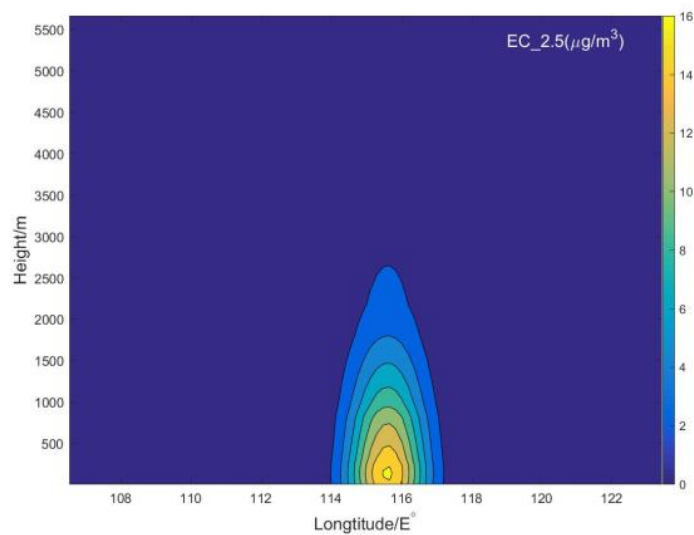

Figure 1 Mass concentrations distribution vertically of the No.1 control variable after data assimilatio

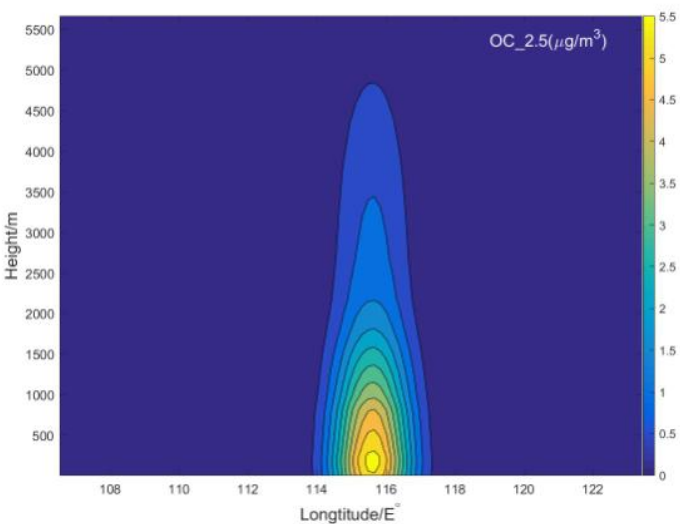

Figure 2 Mass concentrations distribution vertically of the No.2 control variable after data assimilation

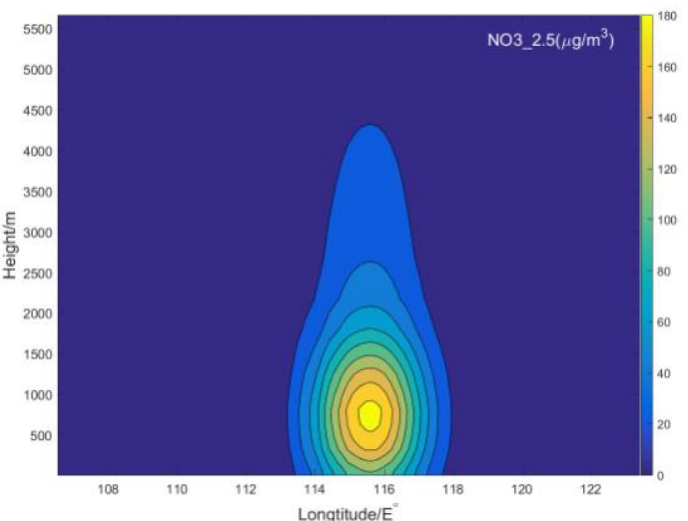

A single-point observation experiment is performed to test the ability of the assimilation scheme. Assume that the mass concentrations of aerosol species in the initial field for the Figure 3 Mass concentrations distribution vertically of the No.3 control variable after data assimilation

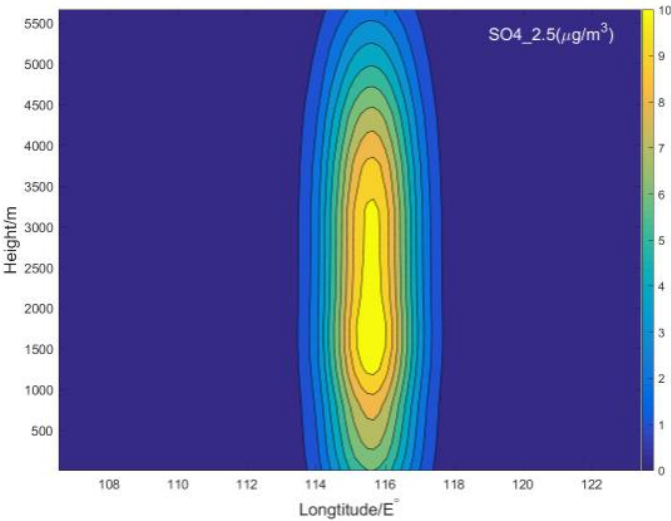

Figure 4 Mass concentrations distribution vertically of the No.4 control variable after data assimilation

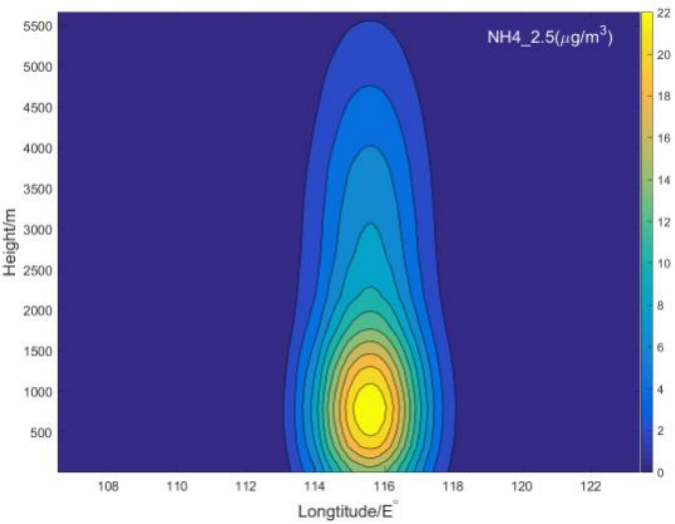

Figure 5 Mass concentrations distribution vertically of the No.7 control variable after data assimilation

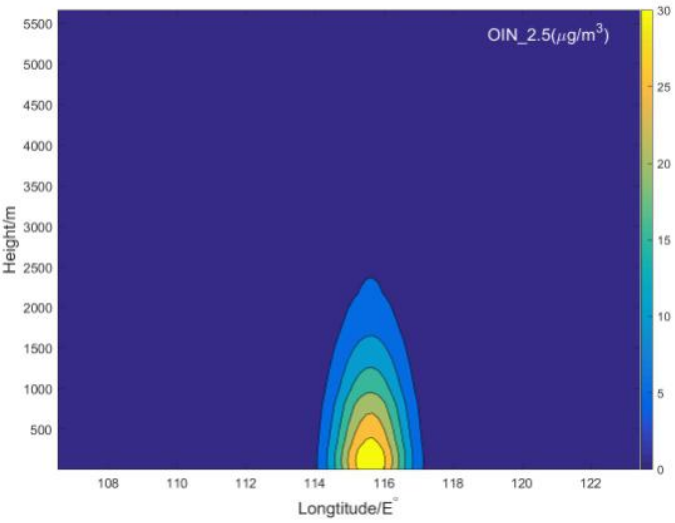

Figure 6 Mass concentrations distribution vertically of the No.8 control variable after data assimilation 
The International Archives of the Photogrammetry, Remote Sensing and Spatial Information Sciences, Volume XLII-3, 2018 ISPRS TC III Mid-term Symposium "Developments, Technologies and Applications in Remote Sensing", 7-10 May, Beijing, China

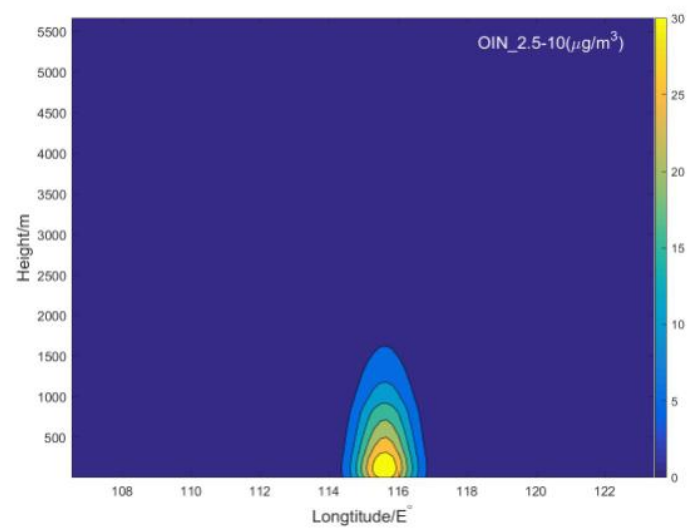

Figure 7 Mass concentrations distribution vertically of the No.16 control variable after data assimilation

\section{References}

Diner, D. J., et al, 2004a. Paragon: an integrated approach for characterizing aerosol climate impacts and environmental interactions. Bulletin of the American Meteorological Society, 85(10), 1491.

Fast, J. D., et al, 2006a. Evolution of ozone, particulates, and aerosol direct radiative forcing in the vicinity of houston using a fully coupled meteorology-chemistry-aerosol model. Journal of Geophysical Research Atmospheres, 111(D21).

Grell, G. A., et al, 2005a. Fully coupled 'online' chemistry within the wrf model. Atmospheric Environment, 39(37), pp.6957-6975.

Jazwinski, A. H. (1970). Stochastic processes and filtering theory. Academic Press.

Kim, Y. J., et al, 2006a. Fine particulate matter characteristics and its impact on visibility impairment at two urban sites in korea: seoul and incheon. Atmospheric Environment, 40(2), pp.593-605.

Li, Z., et al, 2008a. A three-dimensional variational data assimilation scheme for the regional ocean modeling system: implementation and basic experiments. Journal of Geophysical Research Oceans, 113(C6).

Li, Z., et al, 2013a. A three-dimensional variational data assimilation system for multiple aerosol species with wrf/chem and an application to pm2.5 prediction. Atmospheric Chemistry \& Physics Discussions, 13(8), pp. 4265-4278.

Pitchford, M., et al. 2007a. Revised algorithm for estimating light extinction from improve particle speciation data. Journal of the Air \& Waste Management Association, 57(11), 1326.

Zaveri, R. A., et al, 2008a. Model for simulating aerosol interactions and chemistry (mosaic). Journal of Geophysical Research Atmospheres, 113(D13). 